\title{
Eocene temperature gradients
}

To the Editor - Sze Ling Ho and Thomas Laepple ${ }^{1}$ argue that the TEX $_{86}$ palaeothermometer should be calibrated to deep subsurface ocean temperature and that doing so resolves a discrepancy between data and climate model simulations for the early Eocene. Here we argue that their proposed calibration of $\mathrm{TEX}_{86}$ is incompatible with ecological evidence and inappropriate for the largely shallow-water Eocene data. In addition, early Eocene $\mathrm{TEX}_{86}$ data agree reasonably well with other proxy data, such that warm poles and a flat meridional temperature gradient are not unique to $\mathrm{TEX}_{86}$.

The primary assumption behind Ho and Laepple's reinterpretation of Eocene TEX $_{86}$ data is that the Thaumarchaeotal lipids (known as GDGTs) that comprise the proxy are derived from $0-1,000 \mathrm{~m}$ water depth. However, microbial, ecological and oceanographic observations indicate that the sedimentary TEX $_{86}$ signal predominantly represents the shallow subsurface (about $50-300 \mathrm{~m}$ ). This is where maxima in intact polar GDGT and Thaumarchaeotal gene (16S rRNA and amoA) abundances occur ${ }^{2,3}$, consistent with their ecological niche as ammonium oxidizers. Although Thaumarchaeota exist in deeper waters, their cell numbers decline sharply below $\sim 300 \mathrm{~m}$ (ref. 3) and export to the sediment is less efficient ${ }^{4}$. Hence, sedimentary TEX $_{86}$ values closely match those of the upper water column, indicating preservation of an upper-ocean signal ${ }^{4}$.

This evidence justifies several approaches to $\mathrm{TEX}_{86}$ calibration. Despite the Thaumarchaeotal niche in the shallow subsurface, $\mathrm{TEX}_{86}$ can be used as a seasurface temperature (SST) proxy due to the high correlation between subsurface temperatures and SSTs ${ }^{5}$. Although the assumption that surface and subsurface temperatures maintain similar variability through time can be violated, it is reasonable for inference of long-term mean SSTs. A similar assumption is made in $\delta^{18} \mathrm{O}$ palaeothermometry, which relies on foraminifera that reside throughout the upper mixed layer. Shallow subsurface (0-200 m) instead of surface calibrations for $\mathrm{TEX}_{86}$ are also appropriate and have been applied ${ }^{5,6}$. Laboratory mesocosm experiments independently confirm a TEX $_{86}$-temperature sensitivity similar

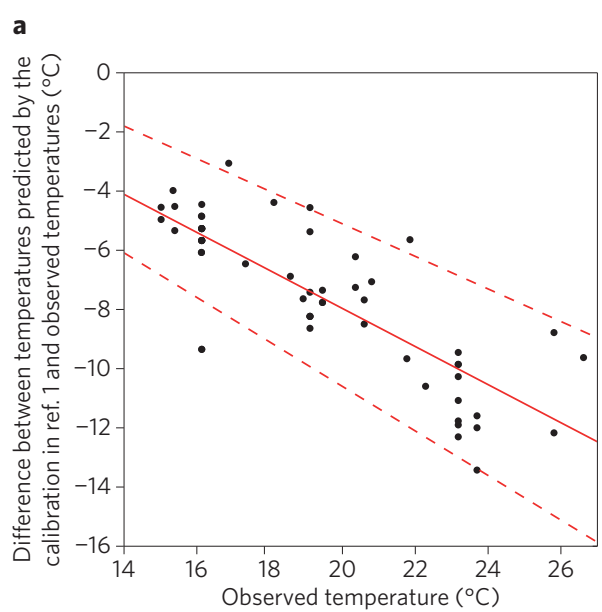

b

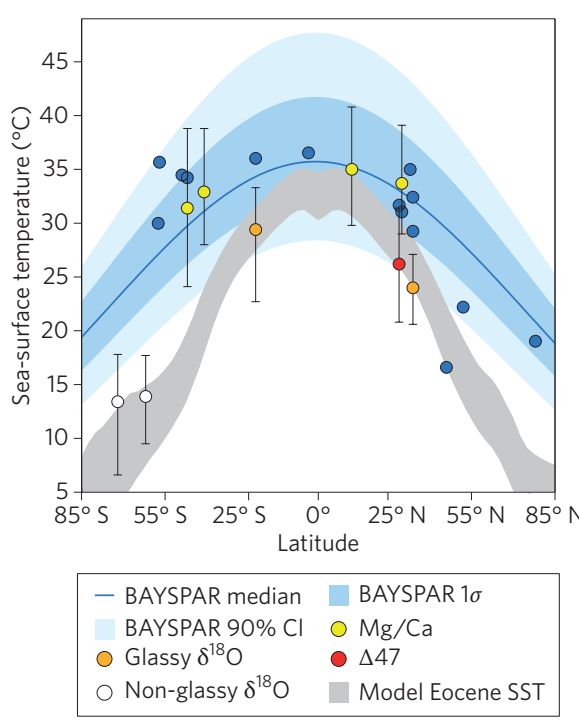

Figure 1 | Bias in the calibration by Ho and Laepple ${ }^{1}$ and Eocene temperature reconstructions. $\mathbf{a}$, In warm $\left(>15^{\circ} \mathrm{C}\right)$ and shallow $(<200 \mathrm{~m})$ sites in the modern ocean, the Ho and Laepple calibration systematically underestimates depth-averaged temperatures (median values presented as points, linear regression as the red line, and maximum and minimum regressions based on the calibration ensemble as dashed red lines). b, Eocene SST predictions based on the BAYSPAR ${ }^{5}$ calibration (blue dots, the median Gaussian fit is shown as a blue line with shading representing $1 \sigma$ and the $90 \%$ $\mathrm{Cl}$ ) are comparable to other proxies ( $90 \% \mathrm{Cl}$ shown) and indicate higher temperatures at mid- to high-latitudes than an ensemble of simulations of early Eocene climate ${ }^{11}$ ( $1 \sigma$ range of SSTs shown as grey shading).

to the surface and shallow subsurface calibrations (slope $\sim 0.015 \mathrm{TEX}_{86}$ units per $\left.{ }^{\circ} \mathrm{C}\right)^{7}$, supporting a shallow-water depth origin for sedimentary GDGTs.

Despite this observational evidence, Ho and Laepple compare the power spectra of paired alkenone $\mathrm{U}_{37}^{\mathrm{K}^{\prime}}$ and $\mathrm{TEX}_{86}$ records, and use this result as the basis of their argument that $\mathrm{TEX}_{86}$ must integrate temperatures to at least $550 \mathrm{~m}$ and possibly as deep as $950 \mathrm{~m}$. The result is a calibration with a $\mathrm{TEX}_{86}$-temperature sensitivity that is twice as large as that found in previous work (slope $\sim 0.030$ ), which is difficult to reconcile with the evidence discussed above. Their approach is flawed in several ways. First, their Fig. 2a demonstrates that all of the variance and explanatory power derives from shallow waters (0-300 $\mathrm{m})$; extending the calibration down to $1,000 \mathrm{~m}$ merely dilutes the regression with relatively invariant data. Second, they assume that $\mathrm{U}_{37}^{\mathrm{K}^{\prime}}$ represents true SST variability, which is unjustified. An appropriate comparison between proxies requires formal modelling of uncertainties associated with both systems, including structural errors relevant to $\mathrm{U}_{37}^{\mathrm{K}^{\prime}}$ such as non-linear temperature sensitivity, diagenetic alteration and lateral advection ${ }^{8}$. Furthermore, even if $\mathrm{U}_{37}^{\mathrm{K}^{\prime}}$ and $\mathrm{TEX}_{86}$ do show different variability in some places, it does not follow that $\mathrm{TEX}_{86}$ is incorrectly calibrated; slight differences in seasonality and depth of production can and do impart variance differences between proxies.

Using their deep-water calibration, Ho and Laepple revisit published Eocene $\mathrm{TEX}_{86}$ data and argue that they can resolve discrepancies between proxies and climate models. We identify two major fallacies in their approach: first, the majority of sites with early Eocene $\mathrm{TEX}_{86}$ data are shallow (water depths between 0 and 200 m; Supplementary Table 1). Applying a calibration that predicts water temperature averaged over a range of $0-550 \mathrm{~m}$ or 0-950 $\mathrm{m}$ is inappropriate. By way of 
example, we apply the Ho and Laepple calibration to warm $\left(T>15^{\circ} \mathrm{C}\right)$, shallow $(0-200 \mathrm{~m})$ sites in the modern surface sediment dataset (see Supplementary Information), which have $\mathrm{TEX}_{86}$ indices analogous to the Eocene values. Their calibration underestimates depth-averaged temperatures by $7.5^{\circ} \mathrm{C}$ in the modern ocean (Fig. 1a), and the underestimation trends with temperature, such that the warmest sites are under-predicted by a greater amount $\left(\sim 12{ }^{\circ} \mathrm{C}\right.$, Fig. 1a). Their calibration will therefore produce an artificially flat gradient between tropical and subtropical temperatures, both in the modern, and in the Eocene, oceans.

Second, TEX $_{86}$-inferred SSTs are not significantly different from those provided by other proxies (Fig. 1b). There is no evidence that $\mathrm{TEX}_{86}$-based temperatures are too high - or produce a gradient that is too flat - relative to other proxies within uncertainties. Only foraminiferal $\delta^{18} \mathrm{O}$ data from Ocean Drilling Program sites 690 and 738 indicate substantially lower temperatures (Fig. 1b); however, these data are probably biased by diagenetic alteration. In the Southwest Pacific, $\mathrm{Mg} / \mathrm{Ca}$ and $\mathrm{TEX}_{86}$ both indicate remarkably high SSTs $\left(\sim 30^{\circ} \mathrm{C}\right)$. These locations may not be representative of their latitudinal band globally; however, even if early Eocene polar temperatures were near $20^{\circ} \mathrm{C}$, as indicated by the Arctic site (Fig. 1b), these are difficult to reproduce with models (Fig. 1b) ${ }^{9}$. Such temperatures are not at all unlikely and are corroborated by independent evidence, such as the presence of palms, baobab and crocodiles at polar latitudes ${ }^{10,11}$. The equable climate problem - the lingering mismatch between proxy data and model simulations - cannot be put to rest.

$\mathrm{TEX}_{86}$ data have played a critical role in constraining early Cenozoic temperatures. Indeed, although we disagree with their approach and conclusions, Ho and Laepple never question the fundamental utility of the proxy. This contrasts with the News \& Views accompanying the article ${ }^{12}$, which suggests that temperature is not the dominant control on $\mathrm{TEX}_{86}$. This view discounts the large body of literature that demonstrates the validity and robustness of the palaeothermometer, as well as the simple fact that ocean temperature explains over $70 \%$ of the variance in modern $\mathrm{TEX}_{86}$ data $^{5} . \mathrm{TEX}_{86}$ has revolutionized our view of past warm climates, and we expect that it will continue to do so.

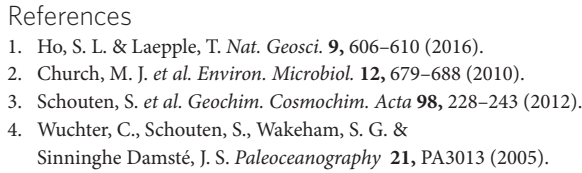

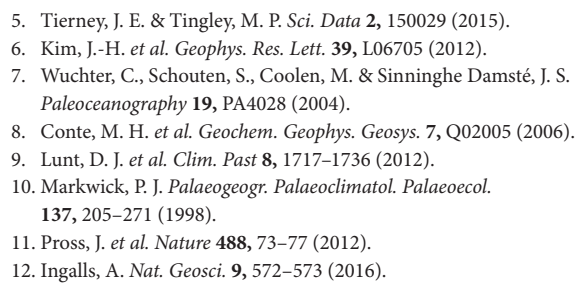

Additional information

Supplementary information is available in the online version of the paper.

Jessica E. Tierney ${ }^{1 \star}$, Jaap S. Sinninghe Damstée, ${ }^{2,3}$ Richard D. Pancost ${ }^{4}$, Appy Sluijs ${ }^{3}$ and James C. Zachos ${ }^{5}$

1 University of Arizona, Department of Geosciences, 1040 E 4th Street, Tucson, Arizona 85721, USA. ${ }^{2}$ NIOZ Royal Netherlands Institute for Sea Research, Department of Marine Microbiology and Biogeochemistry, and University of Utrecht, PO Box 59, 1797 AB

Den Burg, Texel, The Netherlands. ${ }^{3}$ Department of Earth Sciences, Faculty of Geosciences, University of Utrecht, Heidelberglaan 2, 3584C Utrecht, The Netherlands. ${ }^{4}$ Organic Geochemistry Unit, School of Chemistry, Cabot Institute, University of Bristol, Bristol BS8 1TS, UK. ${ }^{5}$ University of California, Santa Cruz, Department of Earth and Planetary Sciences, 1156 High Street, Santa Cruz, California 95064, USA.

*e-mail: jesst@email.arizona.edu

\section{Reply to 'Eocene temperature gradients'}

Sze Ling Ho and Thomas Laepple reply Jessica Tierney and colleagues question the compatibility of our proposed calibration ${ }^{1}$ with ecological evidence. However, our approach was designed with the diverging interpretations of the depth origin of $\mathrm{TEX}_{86}$ in the literature in mind. Contrary to the claim of Tierney et al., we do not a priori assume any depth range but use additional information of past temporal variability to constrain the depth ranges. In the resulting calibration ensemble, the most commonly occurring depths are $100-350 \mathrm{~m}$ (depths in $>80 \%$ of calibration members, full distributions are in the Supplementary Information); this should not be confused with the single calibration with the highest occurrence (0-550 m). This depth range is in agreement with the evidence Tierney et al. summarize. Similarly, the sensitivity derived from mesocosm experiments ${ }^{2,3}\left(52{ }^{\circ} \mathrm{C}\right.$ per unit $\mathrm{TEX}_{86}^{\mathrm{H}}$ in combined datasets $\left.{ }^{4}\right)$ is in fact closer to our estimate $\left(40.8{ }^{\circ} \mathrm{C}\right.$ per unit $\mathrm{TEX}_{86}^{\mathrm{H}}$ ) than to the most commonly applied calibration ${ }^{4}\left(68.4^{\circ} \mathrm{C}\right.$ per unit $\left.\mathrm{TEX}_{86}^{\mathrm{H}}\right)$ or the effective slope of BAYSPAR ${ }^{5}\left(80.8^{\circ} \mathrm{C}\right.$ per unit $\mathrm{TEX}_{86}^{\mathrm{H}}$ ); a result robust to the uncertainty introduced by different $\mathrm{TEX}_{86}$ indices and regression methods.

We argue that the claims made about our statistical analyses do not reflect the evidence presented. Reduced variance and explanatory power of the core-top calibration down the water column prevent an estimation of the slope of the $\mathrm{TEX}_{86}$ calibration and the export depth of Thaumarchaeotal lipids solely from core-top data, which explains the divergence in existing calibrations. We therefore use independent data from the alkenone unsaturation index $\left(\mathrm{U}_{37}^{\mathrm{K}^{\prime}}\right)$ to constrain the slope. The depth habitat of alkenone producers and the temperature sensitivity of the proxy are better constrained than that of $\mathrm{TEX}_{86}$ as demonstrated by agreement between $U_{37}^{K^{\prime}}$ and foraminifera-based proxies ${ }^{6,7}$. Our analysis does include the $\mathrm{U}_{37}^{\mathrm{K}^{\prime}}$ calibration uncertainty and provides independent evidence (Supplementary Fig. 6) that supports the use of $\mathrm{U}_{37}^{\mathrm{K}^{\prime}}$ to constrain $\mathrm{TEX}_{86}$. The differences in seasonal production and export depth between the proxies mentioned by Tierney and colleagues are in fact consistent with our model results. These results demonstrate that when a single or small number of sites are considered, temperature variability - at the sea surface and at different depths tends to differ. However, when averaged across more sites (in our case, 22), the variability at different water depths is expected to converge (Supplementary Fig. 5).

We note that the residuals $\left(6^{\circ} \mathrm{C}\right)$ associated with the application of our calibration to a few modern sites are dominated by sites shallower than $100 \mathrm{~m}$, which have been described as biased towards cold temperatures due to terrestrial input of archaeal lipids. These sites are thus expected to deviate from global calibrations. Notably, even BAYSPAR $^{5}$, which by construction has 